\title{
Photorealistic Computer Graphics Identifying Algorithm based on Edge Detection
}

\author{
Deng lin ${ }^{1, a}$, Liu Changming ${ }^{1, a}$,Li Haiyu ${ }^{1, \text { a }}$ \\ ${ }^{1}$ Computer Department, Air Force Aviation University, changchun 130022, China \\ aemail: gouna@sina.com
}

Keywords: Photorealistic Computer Graphics; natural image; Image Authenticity; SVM

\begin{abstract}
As for the problem the image can be generated by computer software, so that we cannot recognize the authenticity of these images using our eyes. In this paper by analyzing the differences between photorealistic computer graphics and natural image on image texture, we put forward a photorealistic computer graphics identifying algorithm based on edge detection. Image gradient can describe the details of the image and then we extract the feature value according to details of the image. The last we use SVM classifier to classify. The results show that the presented method can not only identify the photorealistic computer graphics and natural image but also have better identification rate.
\end{abstract}

\section{Introduction}

With the rapid development of digital cameras and smart phones, it has great necessary to research image processing technique in the application and operation of recaptured image. It is difficult to identify the natural image and photorealistic computer graphics with the naked eye. The photorealistic computer graphics has given us a tremendous threat. For example the South China tiger incident in 2007 has prompted us that the authenticity of digital images is not only limited to the image of tampering and it is not necessarily true for a digital image. Therefore, it has a very important significance to identify the natural image and photorealistic computer graphics.

In recent years, there are some studies on identification of the photorealistic computer graphics and natural image. Tian-Tsong Ng Etc. presented an method through the geometric characteristics of the image to identify the photorealistic computer graphics and natural image[1]. Farid Etc. presented Higher-order statistical properties the model for image and DCT coefficient histogram class sampling effects[2]. Wu Etc. achieve to identify the photorealistic computer graphics and natural image by difference image histogram features[3]. Li Etc. adopt the Local Binary Pattern to the photorealistic computer graphics and natural image. As for the above problem, by analyzing the differences between the photorealistic computer graphics and natural image generated in the imaging process, we adopt edge detection method to extracting feature values and then take the classification method to identify the photorealistic computer graphics and natural image. The results show that the presented method can not only identify the photorealistic computer graphics and natural image

\section{The Differences Between Photorealistic Computer Graphics and Natural Image}

Natural light will be converted into the RGB Pixels by digital camera CCD sensor. The figure.1 is the process of natural image. In this process, the image is formed by CFA interpolation in digital camera. Due to the complexity of real scenarios and Impact of hardware, the natural image must produce some noise. But the photorealistic computer graphics can be obtained by all kinds of image-editing software. Then these images will be Retouching, sharpening, smoothing by image-editing software. So the photorealistic computer graphics containing the pattern noise is different from the natural image generated by digital cameras. 


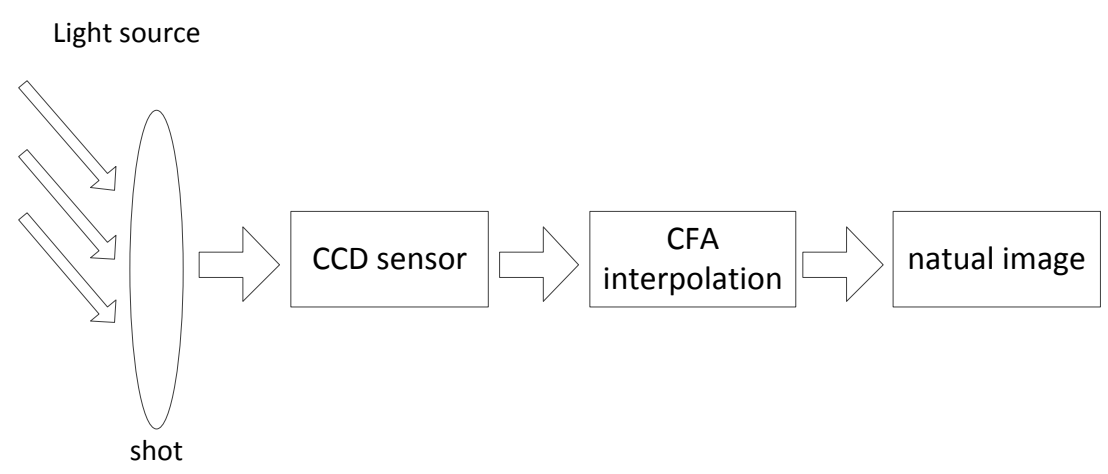

Fig.1. the process of natural image

Due to the current increasingly powerful image editing software, we have too difficult to identify photorealistic computer graphics by eyes. Therefore, we have to judge on the authenticity of these two types by recognition algorithm. In this paper we put forward an identification algorithm based on gradient.

\section{Feature extraction algorithm}

In this paper, the image gradient is calculated to extract eigenvalues. For a continuous function, we can evaluate them first-order partial derivative to solve its rate of change. Using the same idea, the image we also calculate the first order partial derivatives and we calculate the first-order partial derivatives of the $\mathrm{X}$ and $\mathrm{Y}$ directions for the image, as shown in Equation 1.

$$
\left\{\begin{array}{l}
R_{x}(x, y)=\frac{\partial f}{\partial x} \\
R_{y}(x, y)=\frac{\partial f}{\partial y}
\end{array}\right.
$$

For the images, the first order partial derivatives represent the rate of change in the direction of pixels. For smooth area in image, the value of the first order partial derivatives will be relatively small while it will be relatively large in the edge region of the image. The noise is also high-frequency part, and the value of the derivative will be relatively great. In fact, for discrete data such as images, the derivative can be expressed by the gradient, as shown in Equation 2.

$$
\left\{\begin{array}{l}
\frac{\partial f}{\partial x}=f(x+1, y)-f(x, y) \\
\frac{\partial f}{\partial y}=f(x, y+1)-f(x, y)
\end{array}\right.
$$

It is a big problem that the value of the derivative will be a negative. So if we still follow the original gradient values to statistics feature, the dimension of feature vectors will be huge. This will increase the time overhead of the algorithm. In order to solve this problem, we have further improved the algorithm. We extract the maximum gradient value of $\mathrm{X}$ and $\mathrm{Y}$ directions, as shown in Equation 3.

$$
P_{\text {value }}(x, y)=\max \left(\left|R_{x}\right|,\left|R_{y}\right|\right)
$$

Formula (3) shows that, we extract the larger absolute value of the first-order partial derivatives in $\mathrm{X}$ and $\mathrm{Y}$ directions. This can be a good description of the rate of change on image texture. We can get feature image and then we calculate statistical histogram of the feature image to obtain feature vectors. 


\section{Algorithm Framework}

We put forward a photorealistic computer graphics identifying algorithm based on edge detection. The detected image is divided into two parts that are the training and testing. Firstly, we extract the gradient features for image, and then we use the SVM classifier to train samples. Last we use the SVM classifier to test image. As shown in Figure 2:

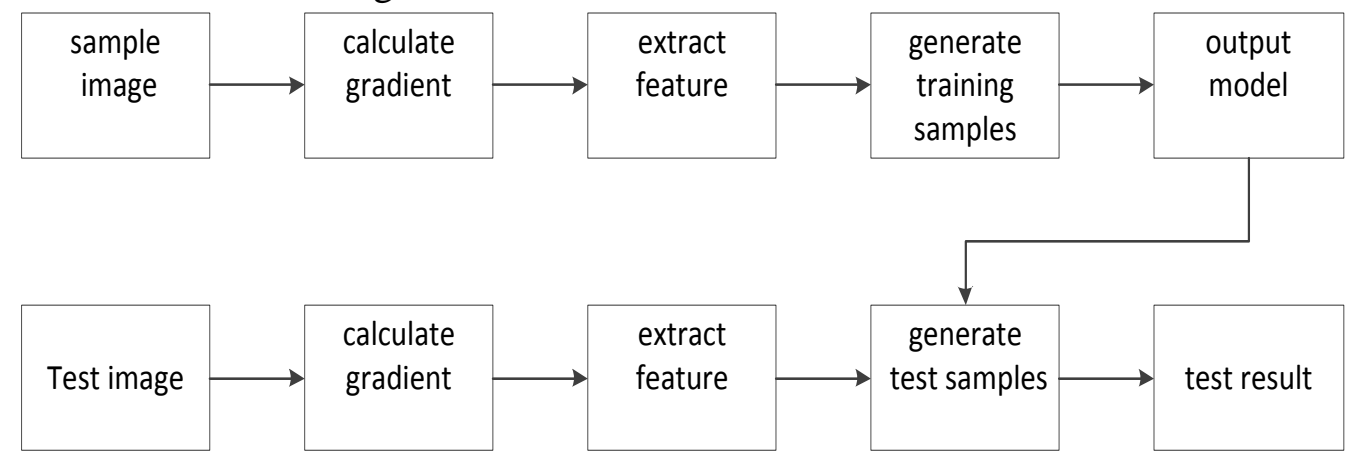

Fig.2. algorithm flow chart

\section{Test Results}

The experimental programming environment is on Microsoft Visual 2010 and the programming language is C language. We use support vector machine (SVM) as classifier. The experiments images are from Columbia University Digital Image Library.In order to the effectiveness of algorithm we take a large number of tests and we get satisfactory results. Every experiment we randomly choose the number of 600 train images and 300 test images.

Table 1 the experimental results and comparison with other algorithm

\begin{tabular}{cc}
\hline algorithm & Accuracy rate \\
\hline Proposed method & $80.30 \%$ \\
Literature 4 & $72.25 \%$ \\
\hline
\end{tabular}

The feature vectors in our algorithm are extracted from the statistical histogram, so these have 256-dimensions vector. In the experimental results, the recognition rate of the algorithm is in line with the actual situation. For more texture information image our algorithm has higher recognition rate while there is lower recognition rate for less texture information image. The image will be affected by the camera CCD sensor and CFA interpolation factors in the imaging process. In particular, there is a strong for the camera when these are taken for the natural image, so the natural images will blur. The algorithm for identifying these images will also be some impact.

\section{Conclusion}

There are so many algorithms to identify photorealistic computer graphics and natural image and these algorithms are relying on the classification. Because of photorealistic computer graphics and natural image having different imaging principle, these have great difference in texture. In this paper we extract gradient feature from photorealistic computer graphics and natural image. The experimental results show that the detection algorithm in this paper is feasible and less dimension feature vectors extracted than those obtained by other algorithms, so the time complexity and space complexity are relatively low. 


\section{References}

[1] Satoshi Kagami,Tomonobu Kitagawa,Koichi Nishiwaki,Tomomichi Sugihara,Masayuki Inaba,Hirochika Inoue. A Fast Dynamically Equilibrated Walking Trajectory Generation Method of Humanoid Robot [J], 2002.

[2] HIROKIK, MINORUA, YASUOK, ET al.RoboCup: a challenge problem for AI and robotics. HirokiK. RoboCup-97: Robot Soccer World Cup [C]. Berlin: Springer, 1998.38-43.

[1] Tian-Tsong Ng, Shih-Fu Chang, Jessie Hsu, LexingXie. Physics-motivated features for distinguishing photographic images and computer graphics[C]. 13th Annual ACM International Conference on Multimedia, 2005: 239- 248.

[2] H.Faridy, S. Lyu. Higher-order wavelet statistics and their application to digital forensics[C]. IEEE Workshop on Statistical Analysis in Computer Vision, 2003:1-8.

[3] WU R, LI X, YANG B. Identifying computer generated graphics via histogram features[C]. 2011 18th IEEE International Conference on Image Processing (ICIP), IEEE, 2011: 1933-1936.

[4] LI Z, YE J, SHI Y Q. Distinguishing computer graphics from photographic images using local binary patterns[M]. Digital Forensics and Watermarking. Springer Berlin Heidelberg, 2013: 228-241.

[5] Tian-Tsong Ng, Shih-Fu Chang. Using geometry invariants for camera response function estimation[C]. IEEE Conference on Computer Vision and Pattern Recognition, 2007: 1-8.

[6] X. Gao1, B. Qiu, J. J. Shen, T. -T. Ng and Y. Q. Shi. A smart phone image database for single image recapture detection[C]. The 9th International Conference on Digital Watermarking, 2010: 90-104.

[7] Bayram S., H. T. Senear and N. Memon, et al. Source Camera Identification Based on CFA Interpolation[C]. Proceedings of IFIP Working Group on Digital Forensics,Orlando, 2006.

[8] Long $\mathrm{Y}_{-}$and Y. Huang. Improvements on Source Camera-Model Identification Based on CFA Interpolation[C]. Proceedings of IEEE 8th Workshop on Multimedia Signal Processing, Canada,2006: 419-424.

[9] Lukas J.,J. Fridrich,ML Goljan. Determining Digital Image Origin Using Sensor Imperfections[C]. Proceedings of SPIE Electronic Imaging, Image and Video Communication and Processing, San Jose: Springer, 2005: 249-260.

[10] Lukas J., J. Fridrich,M. Goljan. Detecting Digital Image Forgeries Using Sensor Pattern Noise[C]. Proceedings of the SPIE International Conference on Security, Steganography, and Watermarking of Multimedia Contents, San Jose: Springer, 2006:362-372.

[11] Hsu Y. F. and S. F. Chang. Detecting Image Splicing Using Geometry Invariants and Camera Characteristics Consistency[C]. International Conference on Multimedia and Expo (ICME), Toronto, 2006.

[12] Mikkilineni A. K‘,P. J. Chiang and G. N. Ali et al. Printer Identification Based on Graylevel Co-occurrence Features for Security and Forensic Application[C]. Proceedings of SPIE International Conference on Security, Steganography and Watermarking of Multimedia Contents, San Jose, 2005. 